\title{
Método de rehabilitación del habla para mejorar la fluidez verbal en la tartamudez infantil
}

\author{
Systematic review. Method of speech rehabilitation to improve verbal fluency \\ in stuttering children
}

Guerrero-Mercado, Lucia '; Martinez-Caceres, Martin *2.

Como citar este artículo: Guerrero-Mercado, Lucia, Martinez-Caceres, Martin. Revisión sistemática. Método de rehabilitación del habla para mejorar la fluidez verbal en la tartamudez infantil. Revistas Signos Fónicos.2018: 4(2).13-28.

Correspondencia autor: luciaguerreromercado@hotmail.com (Lucia Guerrero Mercado)

Recibido: Enero 11, 2018 Aprobado: Marzo 29, 2018.

\begin{abstract}
RESUMEN
INTRODUCCIÓN: a través de una revisión exhaustiva de evidencia científica con esta investigación se pretende establecer cuál es el método más eficiente para mejorar la fluidez verbal en la tartamudez infantil. METODOS: Revisión sistemática utilizando diversas estrategias de búsqueda en diversas bases de datos, contando con una ventana temporal de 10 años. Los estudios incluidos fueron aquellos cuya pesquisa correspondió en seres humanos, población infantil, con indicadores de producción del habla asociadas a la tartamudez y a sus procesos de rehabilitación. RESULTADOS: en la actualidad los profesionales de Fonoaudiología en Latinoamérica están adoptando de forma empírica y basado en la praxis, varias estrategias de rehabilitación para usuarios con alteraciones de la fluidez verbal, diseñadas para rehabilitar el patrón de habla de la tartamudez de la población infantil europea, sin embargo la evidencia científica demuestra la eficiencia y la eficacia de un método de tratamiento denominado programa Lidcombe. ANÁLISIS Y DISCUSIÓN: para el infante es de suma importancia expresar el lenguaje verbal de manera fluida, es ahí donde nace la necesidad del Fonoaudiólogo en buscar estrategias estandarizadas que garanticen la rehabilitación completa de los mecanismos del habla patológica. CONCLUSIONES: el respaldo teórico determina que existen pocos métodos de rehabilitación para la intervención de la tartamudez infantil que sean de fácil acceso para los terapeutas, entre ellos se encontró que existe uno que permite tratar las características del habla de la población infantil australiana y que ha sido aplicado y adaptado en algunos países del continente europeo y asiático.
\end{abstract}

PALABRAS CLAVES: habla, fluidez verbal, rehabilitación, tartamudez, población infantil.

$1 \quad$ *Fonoaudióloga, Magister en Trastornos Cognoscitivos y del Aprendizaje, luciaguerreromercado@hotmail.com, Orcid: 0000-0002-8651-1949, Universidad Manuela Beltrán-Bucaramanga, Colombia.

2 *Estudiante de Fonoaudiología, yesua.oficial@gmail.com,Orcid: 0000-0003-3958-8326. 


\section{ABSTRACT}

INTRODUCTION: through an exhaustive review of scientific evidence, this research aims to establish the most efficient method to improve verbal fluency in childhood stuttering. METHODS: systematic review using various search strategies in various databases, contacting with a 10-year time window; The included studies were those whose research was carried out in human beings, children, with indicators of speech production associated with stuttering and their rehabilitation processes. RESULTS: currently, Speech-Language Professionals in Latin America are adopting the empirical and praxis-based form, various rehabilitation strategies for users with impaired verbal fluency, adapted for the rehabilitation of the speech pattern of population stuttering European infant, however scientific evidence demonstrates the efficiency and effectiveness of a treatment method described in the Lidcombe program. ANALYSIS AND DISCUSSION: for the infant, fluent verbal language is of utmost importance, it is there where the need of the speech pathologist to seek standardized strategies that guarantee the complete rehabilitation of pathological speech mechanisms. CONCLUSIONS: theoretical support determines that there are few rehabilitation methods for the intervention of child stuttering that are easily accessible for therapists, among them will be one that allows treating the speech characteristics of the Australian child population and that has been applied and adapted in some countries on the European and Asian continent.

KEYWORDS: Speech, Stuttering, verbal fluency, rehabilitation, child population.

\section{INTRODUCCIÓN}

La comunicación es donde se materializa el habla como resultado de la articulación de los diferentes procesos motores básicos (respiración, articulación, fonación, masticación y prosodia), el cual le permite al ser humano establecer un contacto lingüístico con el contexto a través de los diferentes actos de habla (ilocutivos, locutivos y perlocutivos), esta interacción favorece el desarrollo del infante como ser biopsicosocial (1). Cuando se presentan características anómalas que alteran la fluidez verbal, con una serie de bloqueos, repeticiones o prolongaciones de silabas, palabras, circunloquios, descoordinación neumofónica y fono articulatoria en el momento de iniciar y mantener una conversación en la emisión del discurso se estaría hablando de disfemia, cabe resaltar que en esta patología también se observa sintomatología no lingüística que se evidencia en el físico de las personas que la padecen, entre ellos: el parpadeo rápido e incluso temblor de los labios, tensión muscular y estado emocional caracterizado por ansiedad (2). Cada uno de estos indicadores de producción del habla asociados a la tartamudez puede ser transitorios o permanentes.

La disfemia suele aparecer desde temprana edad, siendo diagnosticada tartamudez como tal de acuerdo a diversos autores posteriores a los 5 años, ya que el infante, a esta edad ya ha alcanzado por completo el desarrollo fonético-fonológico del lenguaje. Partiendo de esto, en los niños se evidencian dificultades para establecer una interacción oral con su par homologo(3). De esta manera, la presente investigación tiene como objetivo indagar acerca de aquellas estrategias que mejoren la calidad de vida a los infantes que padecen sintomatología relacionada con la disfemia, en pro de brindarles no solo a ellos sino también a los terapeutas en especial los fonoaudiólogos una forma de intervención integral, incluyendo métodos de rehabilitación que permitan guiar hacia un buen pronóstico con respecto a la evolución del trastorno. Además, estas estrategias permitirán dar soporte cuantitativo al rango del proceso rehabilitador del habla de tipo disfemico. Para el desarrollo de esto, se recurrió a la academia para enlazar a través de la investigación el nivel de evidencia científica, dándole valor de uso a lo anterior en el presente trabajo, realizando una búsqueda exhaustiva del respaldo teórico de los diferentes modelos de rehabilitación de la tartamudez infantil, a partir de esto se origina el siguiente interrogante ¿Cuál es el método de rehabilitación del habla más eficiente para mejorar la fluidez verbal en la tartamudez infantil?

\section{MÉTODOS}

Esta es una revisión sistemática de corte descriptivo, puesto que se llevó a cabo la recopilación de evidencia científica. Para lo cual, se tiene en cuenta una ventana temporal de 10 años. La búsqueda de literatura fue realizada en bases de datos indexadas tales como: PUBMED, AJSLP, LSHSS, Jour- 
nal Clinical Linguistics \& Phonetics, International Journal of Speech-Language Pathology, American Journal of Speech-Language Pathology, Science Direct, JSLHR.

Para el diseño de la estructuración de la pregunta en cuestión, se utilizó la estrategia que propone especificar e identificar cuatro componentes que se relacionan a continuación:

P: Población a la que va dirigida (pacientes) especificando grupo de edad, sexo, estadio de la enfermedad, comorbilidad, nivel de riesgos.

I: Intervención que se va evaluar (intervención) que incluye dispositivos, +-procedimientos, pruebas diagnostica, métodos de rehabilitación, exposición, factor pronóstico, factor de riesgos, agentes etiológicos.

C: La evaluación, la intervención, la rehabilitación con la que se quiere (comparar).

O: La variable de resultado o desenlaces de interés (Outcomes), considerando clínicamente relevante para el paciente en el caso de estudios sobre eficacia, pronostico o etiología; y en el caso de los estudios de pruebas diagnostica, estimadores de rendimiento o validez diagnostica (sensibilidad, especificidad, coeficientes de probabilidad y valores predictivos).

Las iniciales de esta denominaciones de los cuatros elementos en ingles dan lugar al acrónimo PICO (patient, intervention, comparison, outcomes) (4). La pregunta de investigación, de la cual nace esta pesquisa se fundamenta bajo criterios de inclusión y exclusión que se categorizan desde la metodología PICO (patient, intervention, comparison, outcomes) de la siguiente manera: P- Tartamudez infantil, I - Rehabilitación y O- Fluidez verbal.

Entre los criterios de inclusión, encontramos la edad (población infantil de 2 años y medio a 5 o 6 años), raza (humana), rehabilitación de la tartamudez y como criterios de exclusión el padecimiento (déficit sensorial o motor del habla), edad (población adulta) y evaluación de la tartamudez. Finalmente, se usa el sistema GRADE que es una herramienta que permite evaluar la calidad de la evidencia y graduar la fuerza de las recomendaciones en el contexto de desarrollo de guías de práctica clínica, revisiones sistemáticas o evaluación de tecnologías sanitarias, con la cual se lleva a cabo el diseño de una tabla de evidencia por desenlaces(5)Assessment, Development, and Evaluation (GRADE.

\section{RESULTADOS}

Se llevó a cabo el diseño de una tabla donde se extrajo información relevante de cada uno de los estudios tenidos en cuenta para la investigación. En la Tabla 1 se extrajo información referente a las variables de año de publicación, nombre del estudio, sitio web y base de datos en la cual fue hallado el artículo. De esta manera, los estudios fueron publicados entre el 2008 y el 2018, que fueron encontrados en los diferentes metas, buscadores mencionados en un principio y tabulando información de 30 estudios. 
TABLA 1. Resultados de la búsqueda en las bases de datos.

\begin{tabular}{|c|c|c|c|}
\hline Año & Nombre & URL & $\begin{array}{l}\text { Base de } \\
\text { Datos }\end{array}$ \\
\hline 2018 & $\begin{array}{l}\text { Comparison of Percentage of Syllables } \\
\text { Stuttered With Parent-Reported Severity } \\
\text { Ratings as a Primary Outcome } \\
\text { Measure in Clinical Trials of } \\
\text { Early Stuttering Treatment (6) }\end{array}$ & $\begin{array}{l}\text { https://www. } \\
\text { ncbi.nlm.nih. } \\
\text { gov/pub- } \\
\text { med/29554191 }\end{array}$ & PUBMED \\
\hline 2018 & $\begin{array}{l}\text { Stuttering in Preschool Children: Direct } \\
\text { Versus Indirect Treatment (7) }\end{array}$ & $\begin{array}{l}\text { https://lshss. } \\
\text { pubs.asha.org/ } \\
\text { article.aspx?arti- } \\
\text { cleid=2669932 }\end{array}$ & $\begin{array}{l}\text { LSHSS } \\
\text { (ASHA) }\end{array}$ \\
\hline 2018 & $\begin{array}{l}\text { The Lidcombe Program and child language } \\
\text { development: Long-term assessment (8) }\end{array}$ & $\begin{array}{l}\text { https://www. } \\
\text { tandfonli- } \\
\text { ne.com/doi/ }\end{array}$ & $\begin{array}{l}\text { Journal } \\
\text { Clinical Lin- } \\
\text { guistics \& } \\
\text { Phonetics }\end{array}$ \\
\hline 2017 & $\begin{array}{l}\text { Evidence for the treatment of co-occurring } \\
\text { stuttering and speech sound disorder: A clinical } \\
\text { case series (9) }\end{array}$ & $\begin{array}{l}\text { https://www. } \\
\text { ncbi.nlm.nih. } \\
\text { gov/pub- } \\
\text { med/28290729 }\end{array}$ & PUBMED \\
\hline 2017 & $\begin{array}{l}\text { Quantification and Systematic Characterization } \\
\text { of Stuttering-Like Disfluencies in Acquired } \\
\text { Apraxia of Speech (10) }\end{array}$ & $\begin{array}{l}\text { https://ajslp. } \\
\text { pubs.asha.org/ } \\
\text { article.aspx?arti- } \\
\text { cleid=2635193 } \\
\end{array}$ & $\begin{array}{l}\text { AJSLP } \\
(\mathrm{ASHA})\end{array}$ \\
\hline 2017 & $\begin{array}{l}\text { The Effects of Parent-Focused Slow Relaxed } \\
\text { Speech Intervention on Articulation Rate, } \\
\text { Response Time Latency, and Fluency } \\
\text { in Preschool Children Who Stutter (11) }\end{array}$ & $\begin{array}{l}\text { https://jslhr. } \\
\text { pubs.asha.org/ } \\
\text { article.aspx?arti- } \\
\text { cleid=2612523 }\end{array}$ & $\begin{array}{l}\text { LSHSS } \\
\text { (ASHA) }\end{array}$ \\
\hline 2016 & $\begin{array}{l}\text { Lidcombe Program Webcam Treatment } \\
\text { for Early Stuttering: A Randomized } \\
\text { Controlled Trial (12) }\end{array}$ & $\begin{array}{l}\text { https://jslhr. } \\
\text { pubs.asha.org/ } \\
\text { article.aspx?arti- } \\
\text { cleid=2553019 }\end{array}$ & $\begin{array}{l}\text { LSHSS } \\
\text { (ASHA) }\end{array}$ \\
\hline 2016 & $\begin{array}{l}\text { Case file audit of Lidcombe program outcomes } \\
\text { in a student-led } \\
\text { stuttering clinic (13) }\end{array}$ & $\begin{array}{l}\text { https://www. } \\
\text { tandfonli- } \\
\text { ne.com/doi/ }\end{array}$ & $\begin{array}{l}\text { Journal } \\
\text { International } \\
\text { Journal of } \\
\text { Speech-Lan- } \\
\text { guage Pa- } \\
\text { thology }\end{array}$ \\
\hline 2016 & $\begin{array}{l}\text { DisordersParent verbal contingencies during } \\
\text { the Lidcombe Program:Observations and sta- } \\
\text { tistical modeling of the treatment process (14) }\end{array}$ & $\begin{array}{l}\text { https://www. } \\
\text { ncbi.nlm.nih. } \\
\text { gov/pub- } \\
\text { med/26897495 }\end{array}$ & PUBMED \\
\hline 2015 & $\begin{array}{l}\text { Economic evaluation of stuttering treatment in } \\
\text { preschool children: The RESTART-study(15) }\end{array}$ & $\begin{array}{l}\text { https://www. } \\
\text { sciencedirect. } \\
\text { com/science/ } \\
\text { article/abs/pii/ }\end{array}$ & $\begin{array}{l}\text { ScienceDi- } \\
\text { rect }\end{array}$ \\
\hline
\end{tabular}

Fuente: Autores. 
Continuación TABLA 1. Resultados de la búsqueda en las bases de datos.

\begin{tabular}{|c|c|c|c|}
\hline Año & Nombre & Url & Base de datos \\
\hline 2015 & $\begin{array}{l}\text { Perspectives of Clinicians Involved in the } \\
\text { RESTART-Study: Outcomes of a Focus } \\
\text { Group (16) }\end{array}$ & $\begin{array}{l}\text { https://ajslp.pubs.asha.org/article. } \\
\text { aspx?articleid=2442494 }\end{array}$ & LSHSS (ASHA) \\
\hline 2015 & $\begin{array}{l}\text { Predicting Treatment Time and Long-Term } \\
\text { Outcome of the Lidcombe Program: A Rep- } \\
\text { lication and Reanalysis (17)2009. }\end{array}$ & $\begin{array}{l}\text { https://ajslp.pubs.asha.org/article. } \\
\text { aspx?articleid=2381652 }\end{array}$ & LSHSS (ASHA) \\
\hline 2015 & $\begin{array}{l}\text { An Overview of } \quad \text { Managing } \\
\text { Stuttering in Japan (18). }\end{array}$ & $\begin{array}{l}\text { https://ajslp.pubs.asha.org/article. } \\
\text { aspx?articleid=1890535 }\end{array}$ & AJSLP (ASHA) \\
\hline 2014 & $\begin{array}{l}\text { Group Lidcombe Program Treatment for } \\
\text { Early Stuttering: A Randomized Controlled } \\
\text { Trial (19). }\end{array}$ & $\begin{array}{l}\text { https://jslhr.pubs.asha.org/article. } \\
\text { aspx?articleid=1873037 }\end{array}$ & LSHSS (ASHA) \\
\hline 2014 & $\begin{array}{l}\text { Webcam Delivery of the Lidcombe Program } \\
\text { for Early Stuttering: A Phase I Clinical Trial } \\
\text { (20) }\end{array}$ & $\begin{array}{l}\text { https://jslhr.pubs.asha.org/article. } \\
\text { aspx?articleid=1828616 }\end{array}$ & LSHSS (ASHA) \\
\hline 2014 & $\begin{array}{l}\text { Effectiveness of the Lidcombe Program for } \\
\text { early stuttering in Australian community } \\
\text { clinics(21) }\end{array}$ & $\begin{array}{l}\text { https://www.ncbi.nlm.nih.gov/ } \\
\text { pubmed/23691980 }\end{array}$ & PUBMED \\
\hline 2013 & $\begin{array}{l}\text { Two-Year Follow-Up of the Lidcombe } \\
\text { Program in Ten Swedish-Speaking Children } \\
\text { (22) }\end{array}$ & $\begin{array}{l}\text { https://www.ncbi.nlm.nih.gov/ } \\
\text { pubmed/23075700 }\end{array}$ & PUBMED \\
\hline 2012 & $\begin{array}{l}\text { Investigating optimal intervention inten- } \\
\text { sity with the Lidcombe Program of early } \\
\text { stuttering intervention ( } 23 \text { ) }\end{array}$ & $\begin{array}{l}\text { https://www.researchgate.net/ } \\
\text { publication/228098959_Investigat- } \\
\text { ing_optimal_intervention_intensi- } \\
\text { ty_with_the_Lidcombe_Program_- } \\
\text { of_early_stuttering_intervention }\end{array}$ & $\begin{array}{l}\text { Internation- } \\
\text { al Journal of } \\
\text { Speech-Lan- } \\
\text { guage Pathol- } \\
\text { ogy }\end{array}$ \\
\hline 2012 & $\begin{array}{l}\text { Evaluación, intervención y evolución en un } \\
\text { caso de tartamudez temprana (24) }\end{array}$ & $\begin{array}{l}\text { https://www.sciencedirect. } \\
\text { com/science/article/pii/ } \\
\text { S1137817411700437 }\end{array}$ & ScienceDirect \\
\hline 2011 & $\begin{array}{l}\text { North-American Lidcombe Program file } \\
\text { audit: Replication and metaanalysis (25) }\end{array}$ & $\begin{array}{l}\text { https://www.ncbi.nlm.nih.gov/ } \\
\text { pubmed/21793775 }\end{array}$ & PUBMED \\
\hline 2011 & $\begin{array}{l}\text { The Lidcombe Program of early stuttering } \\
\text { intervention: mothers' experiences (26) }\end{array}$ & $\begin{array}{l}\text { https://www.sciencedirect. } \\
\text { com/science/article/pii/ } \\
\text { S0094730X10000215 }\end{array}$ & ScienceDirect \\
\hline 2010 & 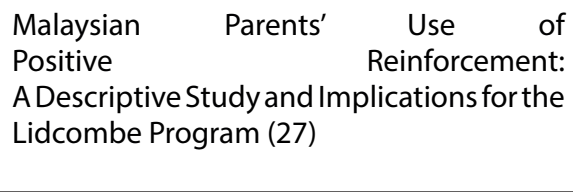 & $\begin{array}{l}\text { https://www.tandfonline.com/doi/ } \\
0.1179 / 136132810805335119\end{array}$ & $\begin{array}{l}\text { Asia Pacif- } \\
\text { ic Journal of } \\
\text { Speech, Lan- } \\
\text { guage and } \\
\text { Hearing }\end{array}$ \\
\hline 2010 & $\begin{array}{l}\text { Follow-up of } 6-10 \text {-year-old stuttering chil- } \\
\text { dren after Lidcombe Program treatment: A } \\
\text { Phase I trial }(28)(2 \text {. }\end{array}$ & $\begin{array}{l}\text { https://www.ncbi.nlm.nih.gov/ } \\
\text { pubmed/20113771 }\end{array}$ & PUBMED \\
\hline 2009 & $\begin{array}{l}\text { Intervention with the Lidcombe } \\
\text { Program for a Bilingual School-Age Child } \\
\text { Who Stutters in Iran (29). }\end{array}$ & $\begin{array}{l}\text { https://www.ncbi.nlm.nih.gov/ } \\
\text { pubmed/19786790 }\end{array}$ & PUBMED \\
\hline
\end{tabular}

Fuente: Autores. 
Continuación TABLA 1. Resultados de la búsqueda en las bases de datos.

\begin{tabular}{|c|c|c|c|}
\hline Año & Nombre & Url & Base de datos \\
\hline 2009 & $\begin{array}{l}\text { Long-Term Outcome of the Lidcombe Pro- } \\
\text { gram for Early Stuttering Intervention (30). }\end{array}$ & $\begin{array}{l}\text { https://ajslp.pubs. } \\
\text { asha.org/article.aspx- } \\
\text { ?articleid=1757602 }\end{array}$ & $\begin{array}{l}\text { AJSLP } \\
\text { (ASHA) }\end{array}$ \\
\hline 2009 & $\begin{array}{l}\text { Stuttering in English-Mandarin Bilingual } \\
\text { Speakers: The Influence of Language Domi- } \\
\text { nance on Stuttering Severity (31). }\end{array}$ & $\begin{array}{l}\text { https://jslhr.pubs. } \\
\text { asha.org/article.aspx- } \\
\text { ?articleid=1775135 }\end{array}$ & $\begin{array}{l}\text { JSLHR } \\
\text { (ASHA) }\end{array}$ \\
\hline 2008 & $\begin{array}{l}\text { Extended follow-up of a randomized } \\
\text { controlled trial of the Lidcombe Program of } \\
\text { Early Stuttering Intervention (32) }\end{array}$ & $\begin{array}{l}\text { https://www. } \\
\text { ncbi.nlm.nih.gov/ } \\
\text { pubmed/18608610 }\end{array}$ & PUBMED \\
\hline 2008 & $\begin{array}{l}\text { A randomized control trial to investigate the } \\
\text { impact of the Lidcombe } \\
\text { Program on early stuttering in Ger- } \\
\text { man-speaking preschoolers (33) }\end{array}$ & $\begin{array}{l}\text { https://www. } \\
\text { ncbi.nlm.nih.gov/ } \\
\text { pubmed/18280869 }\end{array}$ & PUBMED \\
\hline 2008 & $\begin{array}{l}\text { A Phase II Trial of Telehealth Delivery of the } \\
\text { Lidcombe Program of Early Stuttering Inter- } \\
\text { vention (34) }\end{array}$ & $\begin{array}{l}\text { https://www.re- } \\
\text { searchgate.net/pub- } \\
\text { lication/5403079_A_- } \\
\text { Phase_II_Trial_of_- } \\
\text { Telehealth_Deliv- } \\
\text { ery_of_the_Lid- } \\
\text { combe_Program_of_ } \\
\text { Early_Stuttering_In- } \\
\text { tervention }\end{array}$ & $\begin{array}{l}\text { American } \\
\text { Journal of } \\
\text { peech-Lan- } \\
\text { guage } \\
\text { Pathology }\end{array}$ \\
\hline 2008 & $\begin{array}{l}\text { Quantification and Systematic Characteriza- } \\
\text { tion } \\
\text { of Stuttering-Like Disfluencies in Acquired } \\
\text { Apraxia of Speech (15). }\end{array}$ & $\begin{array}{l}\text { https://ajslp.pubs. } \\
\text { asha.org/article.aspx- } \\
\text { ?articleid=2635193 }\end{array}$ & $\begin{array}{l}\text { AjSLP } \\
(\mathrm{ASHA})\end{array}$ \\
\hline
\end{tabular}

\section{Fuente: Autores.}

De acuerdo a los criterios de inclusión y exclusión se distinguen un total de treinta artículos que hacen referencia a método de rehabilitación de la fluidez verbal en niños con tartamudez (Tabla 2).

TABLA 2. Distribución de los estudios encontrados de acuerdo a la base de datos y artículos de inclusión.

\begin{tabular}{cc}
\hline Base de datos & Incluidos \\
\hline Pubmed & 10 \\
\hline AJSLP & 6 \\
\hline LSHSS & 7 \\
\hline $\begin{array}{c}\text { Journal Clinical Linguistics \& Phonetics } \\
\text { International Journal of Speech-Language } \\
\text { Pathology }\end{array}$ & 1 \\
\hline $\begin{array}{c}\text { American Journal of Speech-Language Pa- } \\
\text { thology }\end{array}$ & 1 \\
\hline Science Direct & 1 \\
\hline JSLHR & 3 \\
\hline Total & 1 \\
\hline
\end{tabular}

Fuente: Autores.

Se lleva a cabo la estructuración de una tabla de evidencia, con un total de 17 estudios con diseño metodológico tanto observacional como aleatorio. A nivel general estos estudios fueron evaluados dentro de un rango de calidad de evidencia baja para 4 estudios, moderada para 8 estudios y alta para 5 estudios. Teniendo diferentes consideraciones en cada una de las descripciones hechas a los estudios. Además se evaluá el nivel de importancia, resaltando que 15 de los 17 estudios tuvieron un nivel de importancia "importante" (Ver Tabla 3). 
TABLA 3. Tabla de nivel de evidencia GRADE.

\begin{tabular}{|c|c|c|c|c|c|c|c|c|c|c|c|c|}
\hline \multicolumn{7}{|c|}{ Certainty assessment } & \multicolumn{2}{|c|}{ № de pacientes } & \multicolumn{2}{|c|}{ Efecto } & \multirow[b]{2}{*}{ Certainty } & \multirow[b]{2}{*}{ Importancia } \\
\hline $\begin{array}{l}\text { № de } \\
\text { estudios }\end{array}$ & $\begin{array}{l}\text { Diseño } \\
\text { de es- } \\
\text { tudio }\end{array}$ & $\begin{array}{l}\text { Ries- } \\
\text { go de } \\
\text { sesgo }\end{array}$ & $\begin{array}{l}\text { Incon- } \\
\text { sisten- } \\
\text { cia }\end{array}$ & $\begin{array}{l}\text { E vi- } \\
\text { den- } \\
\text { c i a } \\
\text { indi- } \\
\text { recta }\end{array}$ & $\begin{array}{l}\text { I m - } \\
\text { pre- } \\
\text { c i - } \\
\text { sión }\end{array}$ & $\begin{array}{l}\text { O t r a s } \\
\text { conside- } \\
\text { raciones }\end{array}$ & $\begin{array}{l}\text { El Método } \\
\text { Lidcombe }\end{array}$ & $\begin{array}{l}\text { Mé- } \\
\text { to o- } \\
\text { d o s } \\
\text { más } \\
\text { ef i - } \\
\text { cien- } \\
\text { tes }\end{array}$ & $\begin{array}{l}\text { Rela- } \\
\text { ti vo } \\
(95 \% \\
\text { Cl) }\end{array}$ & $\begin{array}{l}A \text { b - } \\
\text { s o - } \\
\text { I u to } \\
(95 \% \\
\text { Cl) }\end{array}$ & & \\
\hline
\end{tabular}

El método Lidcombe es capaz de adaptarse a las necesidades y diferencias de cada niño y familia individuales (seguimiento: rango 3 años a 6 años : evaluado con : Evidencia de la investigación externa)

\begin{tabular}{|c|c|c|c|c|c|c|c|c|c|c|c|c|}
\hline 1 & $\begin{array}{l}\text { e ns a - } \\
\text { y o s } \\
\text { aleato- } \\
\text { rios }\end{array}$ & $\begin{array}{l}\text { no es } \\
\text { serio }\end{array}$ & $\begin{array}{l}\text { no es } \\
\text { serio }^{\text {a }}\end{array}$ & $\begin{array}{l}\text { no es } \\
\text { serio }\end{array}$ & $\begin{array}{ll}n & o \\
\text { e } & \text { s } \\
\text { serio }\end{array}$ & $\begin{array}{l}\text { fuerte } \\
\text { asocia- } \\
\text { c i ó } n \\
\text { gradiente } \\
\text { de do- } \\
\text { sis-res- } \\
\text { puesta }\end{array}$ & $\begin{array}{l}3 / \\
(100.0 \%)\end{array}$ & $0.0 \%$ & $\begin{array}{c}\text { RR } \\
0.5 \\
(0.3 \mathrm{a} \\
0.7)\end{array}$ & $\begin{array}{c}0 \text { me- } \\
\text { nos } \\
\text { por } \\
100 \\
\text { (de } 0 \\
\text { me- } \\
\text { nos } \\
\text { a } 0 \\
\text { me- } \\
\text { nos) }\end{array}$ & $\bigoplus \bigoplus_{\text {ALTA }} \bigoplus \bigoplus$ & IMPORTANTE \\
\hline
\end{tabular}

Mecanismos subyacentes de tratamiento programa LidcombeNuevo (seguimiento: rango 9 meses a 18 meses; evaluado con : Datos existentes explorando si las reducciones de ING STUTTER)

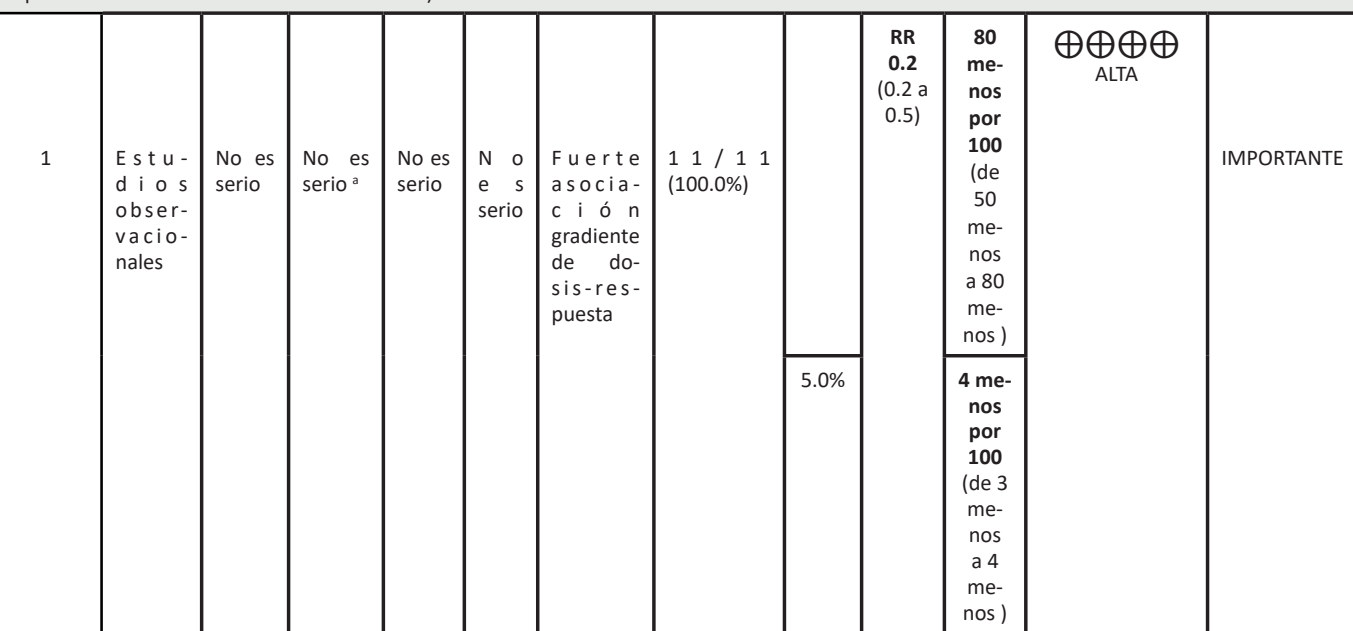

Escasez de pruebas para guiar el tratamiento de los niños que hablan produciendo tartamudeo y trastorno de sonido de voz (evaluado con : Casos clínicos)

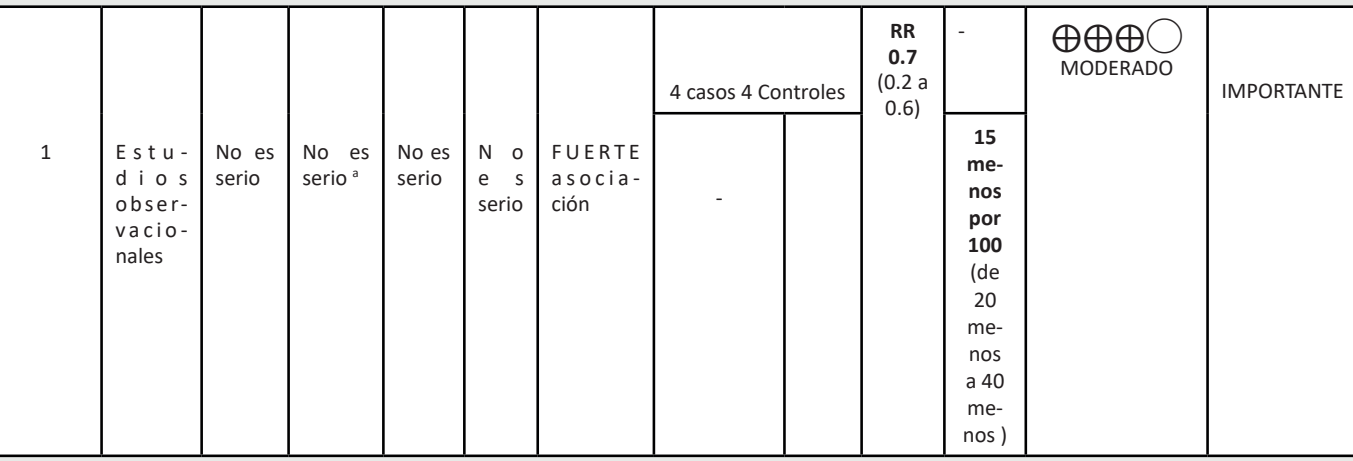

Cuantificar y describir la falta de fluidez de los hablantes con apraxia del habla adquirida (AOS), utilizando Lidcombe (seguimiento: rango 1 semanas a 4 semanas; evaluado con : repetición de palabras de una y varias sílabas a partir de un protocolo y tareas de habla conectadas )

\begin{tabular}{|c|c|c|c|c|c|c|c|c|c|c|c|c|}
\hline 1 & $\begin{array}{l}\text { Estu- } \\
\text { dios } \\
\text { obser- } \\
\text { vacio- } \\
\text { nales }\end{array}$ & $\begin{array}{l}\text { No es } \\
\text { serio }\end{array}$ & $\begin{array}{l}\text { No es } \\
\text { serio }^{\text {a }}\end{array}$ & $\begin{array}{l}\text { No es } \\
\text { serio }\end{array}$ & $\begin{array}{l}\text { No } \\
\text { es } \\
\text { se- } \\
\text { rio }\end{array}$ & ninguno & $\begin{array}{c}20 / 20 \\
(100.0 \%)\end{array}$ & - & - & - & $\bigoplus \bigoplus_{\text {BAJA }} \bigcirc$ & $\begin{array}{l}\text { NO ES IM- } \\
\text { PORTANTE }\end{array}$ \\
\hline
\end{tabular}

Fuente: Autores. 
Continuación TABLA 3. Tabla de nivel de evidencia GRADE.

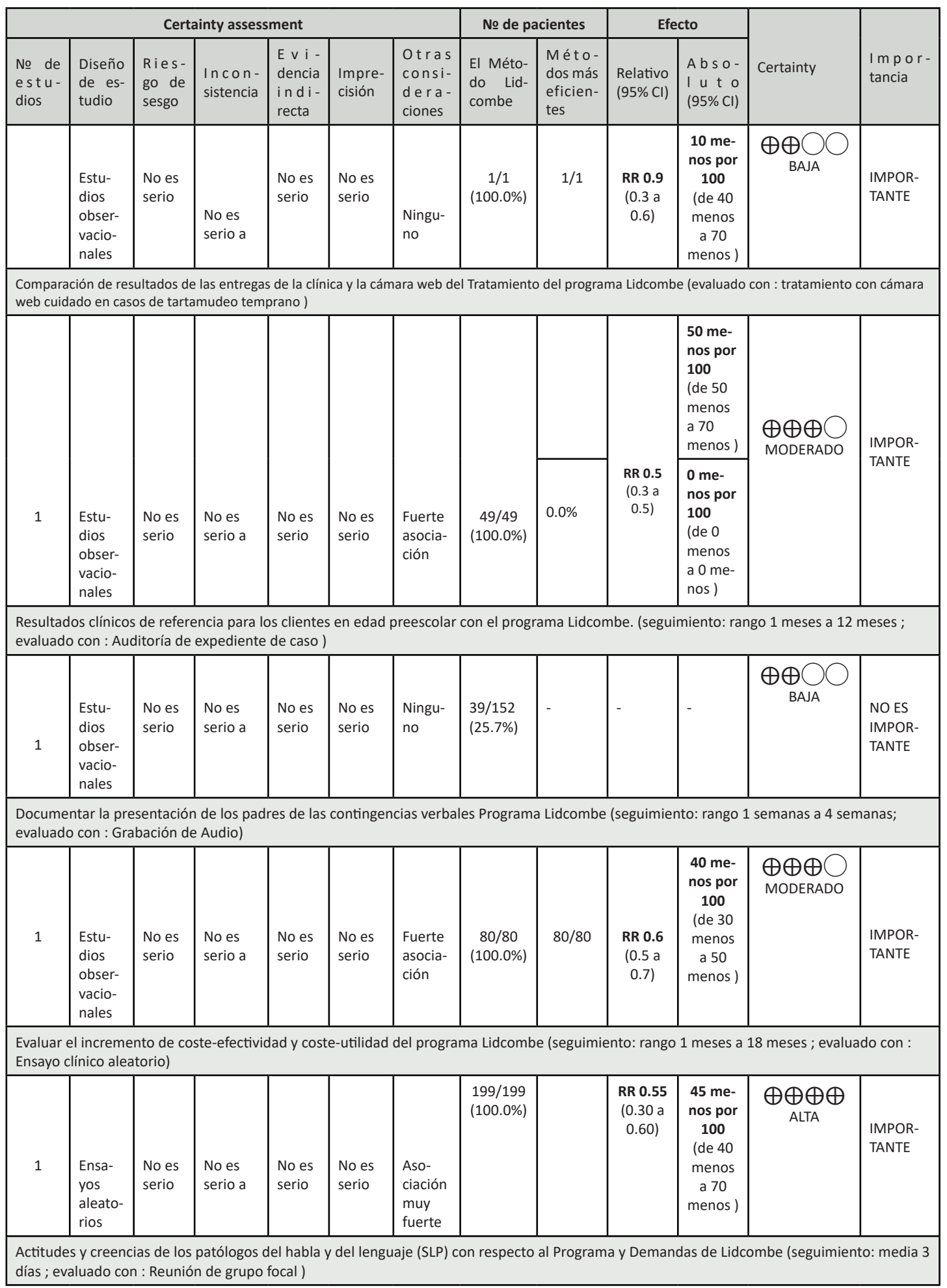

Fuente: Autores. 
Continuación TABLA 3. Tabla de nivel de evidencia GRADE.

\begin{tabular}{|c|c|c|c|c|c|c|c|c|c|c|c|c|}
\hline \multicolumn{7}{|c|}{ Certainty assessment } & \multicolumn{2}{|c|}{ № de pacientes } & \multicolumn{2}{|c|}{ Efecto } & \multirow[b]{2}{*}{ Certainty } & \multirow[b]{2}{*}{$\begin{array}{l}\text { I m p o r - } \\
\text { tancia }\end{array}$} \\
\hline $\begin{array}{l}\text { № } \\
\text { d e } \\
\text { estu- } \\
\text { dios }\end{array}$ & $\begin{array}{l}\text { Diseño } \\
\text { de estu- } \\
\text { dio }\end{array}$ & $\begin{array}{l}\text { Riesgo } \\
\text { de sesgo }\end{array}$ & $\begin{array}{l}\text { Incon- } \\
\text { sisten- } \\
\text { cia }\end{array}$ & $\begin{array}{l}\text { E vi - } \\
\text { de n- } \\
\text { c i a } \\
\text { indi- } \\
\text { recta }\end{array}$ & $\begin{array}{l}\text { I m - } \\
\text { pre- } \\
\text { c i - } \\
\text { sión }\end{array}$ & $\begin{array}{l}\text { Otras } \\
\text { con- } \\
\text { side- } \\
\text { racio- } \\
\text { nes }\end{array}$ & $\begin{array}{l}\text { El Méto- } \\
\text { do Lid- } \\
\text { combe }\end{array}$ & $\begin{array}{l}\text { Métodos } \\
\text { más efi- } \\
\text { cientes }\end{array}$ & $\begin{array}{l}\text { Re la }- \\
\text { t i v o } \\
\text { ( } 95 \% \\
\text { Cl) }\end{array}$ & $\begin{array}{l}\text { A b so- } \\
\text { I u t o } \\
(95 \% \mathrm{Cl})\end{array}$ & & \\
\hline 1 & $\begin{array}{l}\text { Estudios } \\
\text { observa- } \\
\text { cionales }\end{array}$ & $\begin{array}{l}\text { No es } \\
\text { serio }\end{array}$ & $\begin{array}{l}\text { No es } \\
\text { serio a }\end{array}$ & $\begin{array}{l}\text { No es } \\
\text { serio }\end{array}$ & $\begin{array}{l}\text { No } \\
\text { es } \\
\text { se- } \\
\text { rio }\end{array}$ & $\begin{array}{l}\text { Fuer- } \\
\text { te } \\
\text { aso- } \\
\text { cia- } \\
\text { ción }\end{array}$ & $\begin{array}{c}13 / 13 \\
(100.0 \%)\end{array}$ & $\begin{array}{c}13 / 13 \\
(100.0 \%)\end{array}$ & $\begin{array}{c}\text { RR } 0.8 \\
(0.5 \mathrm{a} \\
0.8)\end{array}$ & $\begin{array}{c}20 \text { me- } \\
\text { nos por } \\
100 \\
\text { (de } 20 \\
\text { menos } \\
\text { a } 50 \\
\text { menos) }\end{array}$ & $\underset{\text { MODERADO }}{\bigoplus \bigoplus \bigoplus O}$ & $\begin{array}{l}\text { IMPOR- } \\
\text { TANTE }\end{array}$ \\
\hline \multicolumn{13}{|c|}{$\begin{array}{l}\text { Duración del tratamiento y datos de resultados sobre niños que tartamudean (recientemente tratado por el Programa Lidcombe (seguimiento: rango } 1 \\
\text { años a } 2 \text { años; evaluado con : Combinación de Datos con estudios anteriores) }\end{array}$} \\
\hline 1 & $\begin{array}{l}\text { Estudios } \\
\text { observa- } \\
\text { cionales }\end{array}$ & $\begin{array}{l}\text { No es } \\
\text { serio }\end{array}$ & $\begin{array}{l}\text { No es } \\
\text { serio a }\end{array}$ & $\begin{array}{l}\text { No es } \\
\text { serio }\end{array}$ & $\begin{array}{l}\text { No } \\
\text { es } \\
\text { se- } \\
\text { rio }\end{array}$ & $\begin{array}{l}\text { Nin- } \\
\text { guno }\end{array}$ & $\begin{array}{c}29 / 15 \\
(193.3 \%)\end{array}$ & - & - & - & BAJA & $\begin{array}{l}\text { NO ES } \\
\text { IMPOR- } \\
\text { TANTE }\end{array}$ \\
\hline
\end{tabular}

Evidencia del Programa Lidcombe al comparar el tratamiento individual y grupal de preescolares que tartamudean (seguimiento: rango 9 meses a 18 meses; evaluado con : Ensayo controlado aleatorizado )

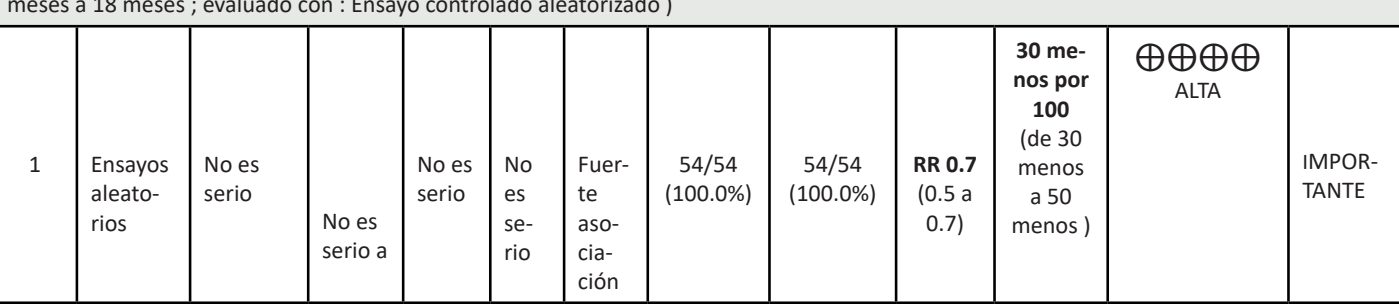

Eficacia potencial, practicidad, y viabilidad de una cámara web de Internet Programa Lidcombe (seguimiento: rango 1 meses a 6 meses ; evaluado con : Tratamiento con cámara web en sus hogares sin clínica)

\begin{tabular}{|c|c|c|c|c|c|c|c|c|c|c|c|c|}
\hline 1 & $\begin{array}{l}\text { Estudios } \\
\text { observa- } \\
\text { cionales }\end{array}$ & $\begin{array}{l}\text { No es } \\
\text { serio }\end{array}$ & $\begin{array}{l}\text { No es } \\
\text { serio a }\end{array}$ & $\begin{array}{l}\text { No es } \\
\text { serio }\end{array}$ & $\begin{array}{l}\text { No } \\
\text { es } \\
\text { se- } \\
\text { rio }\end{array}$ & $\begin{array}{l}\text { Fuer- } \\
\text { te } \\
\text { aso- } \\
\text { cia- } \\
\text { ción }\end{array}$ & $\begin{array}{c}3 / 3 \\
(100.0 \%)\end{array}$ & $\begin{array}{c}3 / 3 \\
(100.0 \%)\end{array}$ & $\begin{array}{c}\text { RR } 0.6 \\
(0.5 \text { a } \\
0.6)\end{array}$ & $\begin{array}{c}40 \text { me- } \\
\text { nos por } \\
100 \\
\text { (de } 40 \\
\text { menos } \\
\text { a } 50 \\
\text { menos) }\end{array}$ & $\bigoplus \bigoplus$ MODERADO & $\begin{array}{l}\text { IMPOR- } \\
\text { TANTE }\end{array}$ \\
\hline
\end{tabular}

Efectividad del programa Lidcombe para el tartamudeo temprano en clínicas comunitarias (seguimiento: rango 1 meses a 9 meses ; evaluado con : Programa Lidcombe)

\begin{tabular}{|c|c|c|c|c|c|c|c|c|c|c|c|c|}
\hline 1 & $\begin{array}{l}\text { Ensayos } \\
\text { aleato- } \\
\text { rios }\end{array}$ & $\begin{array}{l}\text { No es } \\
\text { serio }\end{array}$ & $\begin{array}{l}\text { No es } \\
\text { serio }\end{array}$ & $\begin{array}{l}\text { No es } \\
\text { serio }\end{array}$ & $\begin{array}{l}\text { No } \\
\text { es } \\
\text { se- } \\
\text { rio }\end{array}$ & $\begin{array}{l}\text { Fuer- } \\
\text { te } \\
\text { aso- } \\
\text { cia- } \\
\text { ción }\end{array}$ & $\begin{array}{c}57 / 57 \\
(100.0 \%)\end{array}$ & $\begin{array}{c}57 / 57 \\
(100.0 \%)\end{array}$ & $\begin{array}{c}\text { RR } 0.9 \\
(0.8 \mathrm{a} \\
0.9)\end{array}$ & $\begin{array}{l}10 \text { me- } \\
\text { nos por } \\
100 \\
\text { (de } 10 \\
\text { menos } \\
\text { a } 20 \\
\text { menos) }\end{array}$ & $\underset{\text { ALTA }}{\bigoplus \bigoplus \bigoplus \bigoplus}$ & $\begin{array}{l}\text { IMPOR- } \\
\text { TANTE }\end{array}$ \\
\hline
\end{tabular}

Informar sobre los resultados de 10 niños de habla sueca tratados con el programa Lidcombe (evaluado con : Estudio prospectivo semicontrolada )

Fuente: Autores. 
Continuación TABLA 3. Tabla de nivel de evidencia GRADE.

\begin{tabular}{|c|c|c|c|c|c|c|c|c|c|c|c|c|}
\hline \multicolumn{7}{|c|}{ Certainty assessment } & \multicolumn{2}{|c|}{$\begin{array}{c}\text { № de pacien- } \\
\text { tes }\end{array}$} & \multicolumn{2}{|c|}{ Efecto } & \multirow[b]{2}{*}{ Certainty } & \multirow[b]{2}{*}{$\begin{array}{l}\text { Impor- } \\
\text { tancia }\end{array}$} \\
\hline $\begin{array}{l}\text { № } \\
\text { de estu- } \\
\text { dios }\end{array}$ & $\begin{array}{l}\text { D is e ño } \\
\text { de estu- } \\
\text { dio }\end{array}$ & $\begin{array}{l}\text { Riesgo } \\
\text { de ses- } \\
\text { go }\end{array}$ & $\begin{array}{l}\text { Incon- } \\
\text { sisten- } \\
\text { cia }\end{array}$ & $\begin{array}{l}\text { Eviden- } \\
\text { cia indi- } \\
\text { recta }\end{array}$ & $\begin{array}{l}\text { Impre- } \\
\text { cisión }\end{array}$ & $\begin{array}{l}\text { Otras } \\
\text { consi- } \\
\text { dera- } \\
\text { ciones }\end{array}$ & $\begin{array}{l}\text { E I } \\
\text { M é - } \\
\text { todo } \\
\text { Lid - } \\
\text { com- } \\
\text { be }\end{array}$ & $\begin{array}{l}\text { to - } \\
\text { d o s } \\
\text { m á s } \\
\text { e f i - } \\
\text { cien- } \\
\text { tes }\end{array}$ & $\begin{array}{l}\text { Rela- } \\
\text { t i vo } \\
\text { ( } 95 \% \\
\text { Cl) }\end{array}$ & $\begin{array}{l}A \text { b - } \\
\text { s o - } \\
\text { l u to } \\
(95 \% \\
C I)\end{array}$ & & \\
\hline 1 & $\begin{array}{l}\text { Estudios } \\
\text { observa- } \\
\text { cionales }\end{array}$ & $\begin{array}{l}\text { No es } \\
\text { serio }\end{array}$ & $\begin{array}{l}\text { No es } \\
\text { serio a }\end{array}$ & $\begin{array}{l}\text { No es } \\
\text { serio }\end{array}$ & $\begin{array}{l}\text { No es } \\
\text { serio }\end{array}$ & $\begin{array}{l}\text { Fuerte } \\
\text { asocia- } \\
\text { ción }\end{array}$ & $10 / 10$ & & $\begin{array}{c}\text { RR } 0.8 \\
(0.7 \mathrm{a} \\
0.9)\end{array}$ & $\begin{array}{c}20 \\
\text { me- } \\
\text { nos } \\
\text { por } \\
100 \\
\text { (de } \\
10 \\
\text { me- } \\
\text { nos } \\
\text { a } 30 \\
\text { me- } \\
\text { nos) }\end{array}$ & $\underset{\text { MODERADO }}{\bigoplus \bigoplus \bigoplus \bigoplus}$ & $\begin{array}{l}\text { IMPOR- } \\
\text { TANTE }\end{array}$ \\
\hline
\end{tabular}

Describir el uso de un refuerzo positivo por un pequeño número de padres de Malasia (seguimiento: rango 1 días a 20 días ; evaluado con : Grabación de videos)

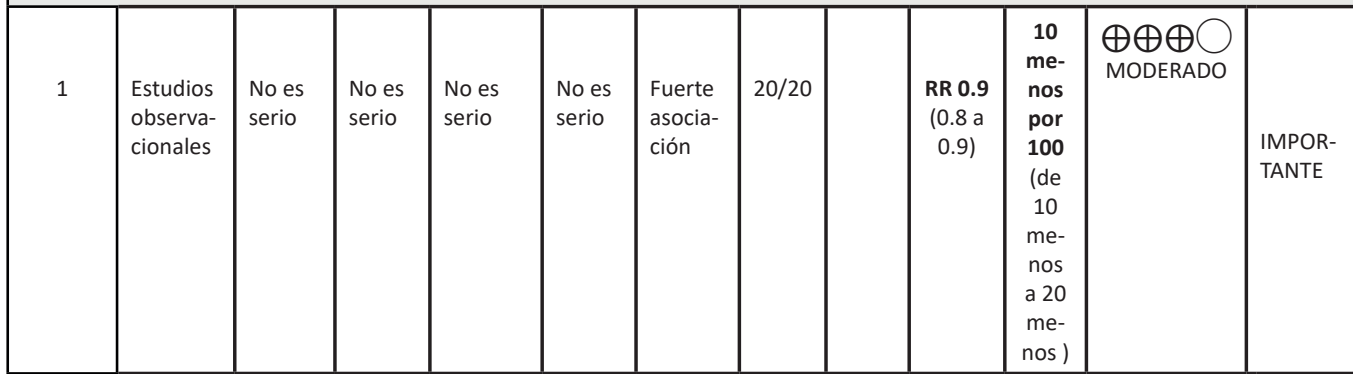

programa Lidcombe y su viabilidad para los niños en edad escolar (seguimiento: rango 1 días a 10 días ; evaluado con : Grabaciones clínica pre-tratamiento de rutina)

\begin{tabular}{|c|c|c|c|c|c|c|c|c|c|c|c|}
\hline 1 & $\begin{array}{l}\text { Estudios } \\
\text { observa- } \\
\text { cionales }\end{array}$ & $\begin{array}{l}\text { No es } \\
\text { serio }\end{array}$ & $\begin{array}{l}\text { No es } \\
\text { serio a }\end{array}$ & $\begin{array}{l}\text { No es } \\
\text { serio }\end{array}$ & $\begin{array}{l}\text { No es } \\
\text { serio }\end{array}$ & $\begin{array}{l}\text { Fuerte } \\
\text { asocia- } \\
\text { ción }\end{array}$ & $12 / 12$ & $\begin{array}{c}\text { RR } \\
0.89 \\
(0.62 \text { a } \\
0.90)\end{array}$ & $\begin{array}{c}10 \\
\text { me- } \\
\text { nos } \\
\text { por } \\
100 \\
\text { (de } 9 \\
\text { me- } \\
\text { nos } \\
\text { a } 35 \\
\text { me- } \\
\text { nos ) }\end{array}$ & MODERADO & $\begin{array}{l}\text { IMPOR- } \\
\text { TANTE }\end{array}$ \\
\hline
\end{tabular}

Fuente: Autores.

Posterior al análisis de los estudios a nivel general, se hizo una recopilación de información procedente de los desenlaces más importantes cuyos resultados guardaban relación con la investigación. De esta manera, se realizó una tabla de evidencia donde se comparaba el método Lidcombe de la disfemia con los métodos más eficientes para la rehabilitación de la fluidez verbal en la tartamudez infantil. De acuerdo a la evidencia científica indagada, los desenlaces variaron su calidad de evidencia entre moderada, baja y alta. La muestra de los estudio estuvo en un rango entre 2 pacientes a 400 pacientes aproximadamente (Tabla 4). 
Tabla 4. Metodología grade, muestra de la evidencia científica del programa LIDCOMBE como el método más eficiente de la fluidez verbal, en la tartamudez infantil.

\begin{tabular}{|c|c|c|c|c|c|}
\hline \multirow[b]{2}{*}{ DESENLACES } & \multicolumn{2}{|c|}{$\begin{array}{c}\text { EFECTOS ABSOLUTOS } \\
\text { ANTICIPADOS * }(95 \% \mathrm{CI})\end{array}$} & \multirow{2}{*}{$\begin{array}{l}\text { EFECTO RELA- } \\
\text { TIVO } \\
(95 \% \mathrm{CI})\end{array}$} & \multirow[t]{2}{*}{$\begin{array}{l}\text { № DE PARTICIPANTES } \\
\text { (ESTUDIOS ) }\end{array}$} & \multirow{2}{*}{$\begin{array}{l}\text { CERTAINTY OF } \\
\text { THE EVIDENCE } \\
\text { (GRADE) }\end{array}$} \\
\hline & $\begin{array}{l}\text { RIESGO } \\
\text { CON MÉ- } \\
\text { TODOS } \\
\text { MÁS } \\
\text { EFICIEN- } \\
\text { TES }\end{array}$ & $\begin{array}{l}\text { RIESGO CON } \\
\text { EL MÉTODO } \\
\text { LIDCOMBE }\end{array}$ & & & \\
\hline \multirow{2}{*}{$\begin{array}{l}\text { El método Lidcombe es capaz de adaptarse a } \\
\text { las necesidades y diferencias de cada niño y } \\
\text { familia individuales evaluado con : Evidencia } \\
\text { de la investigación externa seguimiento: ran- } \\
\text { go } 3 \text { años a } 6 \text { años }\end{array}$} & \multicolumn{2}{|l|}{ Bajo } & $\begin{array}{c}\text { RR } 0.5 \\
(0.3 \text { a } 0.7)\end{array}$ & $\begin{array}{c}3 \\
\text { (1 ECA (experimento } \\
\text { controlado aleatori- } \\
\text { zado)) }\end{array}$ & $\underset{\mathrm{ALTA} a}{\bigoplus \bigoplus \bigoplus}$ \\
\hline & 0 por 100 & $\begin{array}{l}0 \text { por } 100 \\
(0 \text { a } 0)\end{array}$ & & & \\
\hline \multirow{4}{*}{$\begin{array}{l}\text { Mecanismos subyacentes de tratamien- } \\
\text { to programa Lidcombe Nuevo evalua- } \\
\text { do con : Datos existentes exploran- } \\
\text { do si las reducciones de ING STUTTER } \\
\text { seguimiento: rango } 9 \text { meses a } 18 \text { meses }\end{array}$} & \multicolumn{2}{|c|}{ Población estudio } & $\begin{array}{c}\text { RR } 0.2 \\
(0.2 \text { a } 0.5)\end{array}$ & $\begin{array}{c}22 \\
\text { (1 estudio observa- }\end{array}$ & $\bigoplus \bigoplus \bigoplus \bigoplus$ \\
\hline & $\begin{array}{l}100 \text { por } \\
100\end{array}$ & $\begin{array}{c}20 \text { por } 100 \\
(20 \text { a } 50)\end{array}$ & & & \\
\hline & \multicolumn{2}{|l|}{ Bajo } & & & \\
\hline & 5 por 100 & $\begin{array}{l}1 \text { por } 100 \\
(1 \text { a } 3)\end{array}$ & & & \\
\hline \multirow{2}{*}{$\begin{array}{l}\text { Escasez de pruebas para guiar el tratamiento } \\
\text { de los niños que hablan produciendo tarta- } \\
\text { mudeo y trastorno de sonido de voz evalua- } \\
\text { do con: Casos clínicos. }\end{array}$} & Moderado & & $\begin{array}{c}\text { RR } 0.7 \\
(0.2 \text { a } 0.6)\end{array}$ & $\begin{array}{l}4 \text { casos } 4 \text { Controles } \\
\text { (1 estudio observa- } \\
\text { cional ) }\end{array}$ & $\underset{\text { MODERADO a }}{\bigoplus \bigoplus \bigoplus \bigcirc}$ \\
\hline & $\begin{array}{l}50 \text { por } \\
100\end{array}$ & $\begin{array}{c}35 \text { por } 100 \\
(10 \text { a } 30)\end{array}$ & & & \\
\hline $\begin{array}{l}\text { Cuantificar y describir la falta de flui- } \\
\text { dez de los hablantes con apraxia del ha- } \\
\text { bla adquirida (AOS), utilizando Lidcombe } \\
\text { evaluado con: repetición de palabras de } \\
\text { una y varias sílabas a partir de un pro- } \\
\text { tocolo y tareas de habla conectadas } \\
\text { seguimiento: rango } 1 \text { semanas a } 4 \text { semanas. }\end{array}$ & \multicolumn{2}{|l|}{-} & \multicolumn{2}{|r|}{$\begin{array}{l}20 \\
\text { o observacional ) }\end{array}$} & BAJA a \\
\hline $\begin{array}{l}\text { Efectos de una intervención para redu- } \\
\text { cir las tasas de articulación de los cui- } \\
\text { dadores con niños que tartamudean } \\
\text { evaluado con : Estudio grupal de tratamiento } \\
\text { seguimiento: media } 2 \text { días }\end{array}$ & $\begin{array}{l}100 \text { por } \\
100\end{array}$ & $\begin{array}{c}90 \text { por } 100 \\
(30 \text { a } 60)\end{array}$ & $\begin{array}{c}\text { RR } \\
\mathbf{0 . 9} \\
(0.3 \text { a } \\
0.6)\end{array}$ & $\begin{array}{c}2 \\
\text { udio observacional ) }\end{array}$ & $\bigoplus \bigoplus \underset{\text { BAJA a }}{\bigoplus}$ \\
\hline \multirow{4}{*}{$\begin{array}{l}\text { Comparación de resultados de las en- } \\
\text { tregas de la clínica y la cámara web del } \\
\text { Tratamiento del programa Lidcombe } \\
\text { evaluado con : tratamiento con cámara web } \\
\text { cuidado en casos de tartamudeo temprano }\end{array}$} & \multicolumn{2}{|c|}{ Población estudio } & $\begin{array}{c}\text { RR } 0.5 \\
(0.3 \text { a } 0.5)\end{array}$ & $\begin{array}{l}149 \\
(1 \text { estudio observa- } \\
\text { cional ) }\end{array}$ & $\underset{\text { MODERADO a }}{\bigoplus \bigoplus \bigoplus \bigcirc}$ \\
\hline & $\begin{array}{c}100 \text { por } \\
100\end{array}$ & $\begin{array}{c}50 \text { por } \\
100 \\
(30 \text { a } 50)\end{array}$ & & & \\
\hline & Bajo & & & & \\
\hline & $\begin{array}{l}0 \text { por } \\
100\end{array}$ & $\begin{array}{c}0 \text { por } 100 \\
(0 \text { a } 0)\end{array}$ & & & \\
\hline
\end{tabular}

Fuente: Autores. 
Continuación Tabla 4. Metodología grade, muestra de la evidencia científica del programa LIDCOMBE como el método más eficiente de la fluidez verbal, en la tartamudez infantil.

\begin{tabular}{|c|c|c|c|c|c|}
\hline \multirow[t]{2}{*}{ DESENLACES } & \multicolumn{2}{|c|}{$\begin{array}{c}\text { EFECTOS ABSOLUTOS ANTICIPADOS } \\
*(95 \% \mathrm{Cl})\end{array}$} & \multirow{2}{*}{$\begin{array}{l}\text { EFECTO RELA- } \\
\text { TIVO } \\
(95 \% \mathrm{CI})\end{array}$} & \multirow{2}{*}{$\begin{array}{l}\text { № DE PARTI- } \\
\text { CIPANTES } \\
\text { (ESTUDIOS) }\end{array}$} & \multirow{2}{*}{$\begin{array}{l}\text { CERTAINTY } \\
\text { OF THE EVI- } \\
\text { DENCE } \\
\text { (GRADE) }\end{array}$} \\
\hline & $\begin{array}{l}\text { RIESGO CON } \\
\text { MÉTODOS MÁS } \\
\text { EFICIENTES }\end{array}$ & $\begin{array}{l}\text { RIESGO CON EL } \\
\text { MÉTODO LID- } \\
\text { COMBE }\end{array}$ & & & \\
\hline $\begin{array}{l}\text { Resultados clínicos de referencia } \\
\text { para los clientes en edad preescolar } \\
\text { con el programa Lidcombe. evaluado } \\
\text { con: Auditoría de expediente de caso } \\
\text { seguimiento: rango } 1 \text { meses a } 12 \text { meses. }\end{array}$ & Bajo & - & - & $\begin{array}{l}152 \\
\text { (1 estudio ob- } \\
\text { servacional ) }\end{array}$ & $\bigoplus \bigoplus \underset{\text { BAJA }}{\oplus}$ \\
\hline $\begin{array}{l}\text { Documentar la presentación de los padres } \\
\text { de las contingencias verbales Programa } \\
\text { Lidcombe evaluado con: Grabación de } \\
\text { Audio seguimiento: rango } 1 \text { semanas a } 4 \\
\text { semanas. }\end{array}$ & 100 por 100 & $\begin{array}{c}60 \text { por } 100 \\
(50 \text { a } 70)\end{array}$ & $\begin{array}{c}\text { RR } 0.6 \\
(0.5 \text { a } 0.7)\end{array}$ & $\begin{array}{l}160 \\
\text { (1 estudio ob- } \\
\text { servacional ) }\end{array}$ & $\underset{\text { MODERA }}{\bigoplus \bigoplus \bigoplus}$ \\
\hline $\begin{array}{l}\text { Evaluar el incremento de coste-efectividad } \\
\text { y coste-utilidad del programa Lidcombe } \\
\text { evaluado con: Ensayo clínico aleatorio } \\
\text { seguimiento: rango } 1 \text { meses a } 18 \text { meses. }\end{array}$ & 100 por 100 & $\begin{array}{c}55 \text { por } 100 \\
(30 \text { a } 60)\end{array}$ & $\begin{array}{c}\text { RR } 0.55 \\
(0.30 \text { a } 0.60)\end{array}$ & $\begin{array}{l}398 \\
\text { (1 ECA (ex- } \\
\text { perimento } \\
\text { controlado } \\
\text { aleatorizado)) }\end{array}$ & $\underset{\text { ALTA a }}{\bigoplus \bigoplus \bigoplus \bigoplus ~}$ \\
\hline $\begin{array}{l}\text { Actitudes y creencias de los patólogos } \\
\text { del habla y del lenguaje (SLP) con respec- } \\
\text { to al Programa y Demandas de Lidcombe } \\
\text { evaluado con: Reunión de grupo focal } \\
\text { seguimiento: media } 3 \text { días. }\end{array}$ & 100 por 100 & $\begin{array}{c}80 \text { por } 100 \\
(50 \text { a } 80)\end{array}$ & $\begin{array}{c}\text { RR } 0.8 \\
(0.5 \text { a } 0.8)\end{array}$ & $\begin{array}{l}26 \\
\text { (1 estudio ob- } \\
\text { servacional) }\end{array}$ & $\underset{\text { MODERADO a }}{\bigoplus \bigoplus \bigoplus O}$ \\
\hline $\begin{array}{l}\text { Duración del tratamiento y datos de re- } \\
\text { sultados sobre niños que tartamudean } \\
\text { (recientemente tratado por el Progra- } \\
\text { ma Lidcombe evaluado con: Combina- } \\
\text { ción de Datos con estudios anteriores } \\
\text { seguimiento: rango } 1 \text { años a } 2 \text { años. }\end{array}$ & & - & - & $\begin{array}{l}15 \\
\text { (1 estudio ob- } \\
\text { servacional ) }\end{array}$ & $\bigoplus \bigoplus \bigoplus_{\text {BAJA a }} \bigcirc$ \\
\hline $\begin{array}{l}\text { Evidencia del Programa Lidcombe al } \\
\text { comparar el tratamiento individual y gru- } \\
\text { pal de preescolares que tartamudean } \\
\text { evaluado con: ensayo controlado aleato- } \\
\text { rizado seguimiento: rango } 9 \text { meses a } 18 \\
\text { meses. }\end{array}$ & 100 por 100 & $\begin{array}{c}70 \text { por } 100 \\
(50 \text { a } 70)\end{array}$ & $\begin{array}{c}\text { RR } 0.7 \\
(0.5 \text { a } 0.7)\end{array}$ & $\begin{array}{l}108 \\
\text { (1 ECA (ex- } \\
\text { perimento } \\
\text { controlado } \\
\text { aleatorizado)) }\end{array}$ & $\bigoplus \underset{\text { ALTA a }}{\bigoplus \bigoplus \bigoplus}$ \\
\hline $\begin{array}{l}\text { Eficacia potencial, practicidad, y viabilidad } \\
\text { de una cámara web de Internet Programa } \\
\text { Lidcombe evaluado con: tratamiento con } \\
\text { cámara web en sus hogares sin clínica se- } \\
\text { guimiento: rango } 1 \text { meses a } 6 \text { meses. }\end{array}$ & 100 por 100 & $\begin{array}{c}60 \text { por } 100 \\
(50 \text { a } 60)\end{array}$ & $\begin{array}{c}\text { RR } 0.6 \\
(0.5 \text { a } 0.6)\end{array}$ & $\begin{array}{c}6 \\
\text { (1 estudio ob- } \\
\text { servacional ) }\end{array}$ & $\begin{array}{c}\bigoplus \bigoplus \bigoplus \bigcirc \\
\begin{array}{c}\text { MODERA- } \\
\text { DO }^{\mathrm{a}}\end{array}\end{array}$ \\
\hline $\begin{array}{l}\text { Efectividad del programa Lidcombe para el } \\
\text { tartamudeo temprano en clínicas comuni- } \\
\text { tarias evaluado con: Programa Lidcombe } \\
\text { seguimiento: rango } 1 \text { meses a } 9 \text { meses. }\end{array}$ & 100 por 100 & $\begin{array}{c}90 \text { por } 100 \\
(80 \text { a } 90)\end{array}$ & $\begin{array}{c}\text { RR } 0.9 \\
(0.8 \text { a } 0.9)\end{array}$ & $\begin{array}{l}114 \\
\text { (1 ECA (ex- } \\
\text { perimento } \\
\text { controlado } \\
\text { aleatorizado)) }\end{array}$ & $\underset{\text { ALTA }}{\bigoplus \bigoplus \bigoplus}$ \\
\hline $\begin{array}{l}\text { Informar sobre los resultados de } 10 \text { niños } \\
\text { de habla sueca tratados con el programa } \\
\text { Lidcombe evaluado con: estudio prospec- } \\
\text { tivo semicontrolada. }\end{array}$ & 100 por 100 & $\begin{array}{l}80 \text { por } 100 \\
(70 \text { a } 90)\end{array}$ & $\begin{array}{l}\text { RR } 0.8 \\
(0.7 \text { a } 0.9)\end{array}$ & $\begin{array}{l}20 \text { ( } 1 \text { estu } \\
\text { dio obser- } \\
\text { vacional) }\end{array}$ & $\underset{\text { MODERADO a }}{\bigoplus \bigoplus \bigoplus \bigcirc}$ \\
\hline $\begin{array}{l}\text { Describir el uso de un refuerzo positivo por } \\
\text { un pequeño número de padres de Malasia } \\
\text { evaluado con: Grabación de videos segui- } \\
\text { miento: rango } 1 \text { días a } 20 \text { días. }\end{array}$ & 100 por 100 & $\begin{array}{l}90 \text { por } 100 \\
(80 \text { a } 90)\end{array}$ & $\begin{array}{c}\text { RR } 0.9 \\
(0.8 \text { a } 0.9)\end{array}$ & $\begin{array}{c}40 \\
\text { (1 estudio } \\
\text { observa- } \\
\text { cional) }\end{array}$ & $\begin{array}{l}\bigoplus \bigoplus \bigoplus C \\
\text { MODE- } \\
\text { RADO }\end{array}$ \\
\hline $\begin{array}{l}\text { Programa Lidcombe y su viabili- } \\
\text { dad para los niños en edad escolar } \\
\text { evaluado con: Grabaciones clí- } \\
\text { nica pre-tratamiento de rutina } \\
\text { seguimiento: rango } 1 \text { días a } 10 \text { días. }\end{array}$ & 92 por 100 & $\begin{array}{c}82 \text { por } 100 \\
(57 \text { a } 83)\end{array}$ & $\begin{array}{c}\text { RR } 0.89 \\
(0.62 \text { a } 0.90)\end{array}$ & $\begin{array}{c}24 \\
\text { (1 estudio } \\
\text { observa- } \\
\text { cional) }\end{array}$ & $\underset{\text { MODERADO a }}{\bigoplus \bigoplus \bigoplus O}$ \\
\hline
\end{tabular}

Fuente: Autores. 


\section{ANÁLISIS Y DISCUSIÓN}

La disfemia suele aparecer entre los 2 años y medio y los 4 años de edad siendo un trastorno del lenguaje y del habla, que dificulta la comunicación. Es decir, dificulta la recepción y transmisión de mensajes tanto a sus pares como demás individuos. Comúnmente es denominada como tartamudez, trastorno caracterizado por interrupciones de la fluidez e inteligibilidad del habla, repeticiones de sonidos, palabras o frases, o prolongaciones y, en ocasiones, bloqueos (5).

Estas alteraciones iniciales no son voluntarias ni son conductas aprendidas, aunque el esfuerzo y la omisión del habla, que suelen aparecer posteriormente a lo largo de la evolución del problema, sí pueden ser aprendidas. Es importante recalcar que el niño tiene mayor prevalencia para presentar este problema en la etapa preescolar, En general, el riesgo más alto se encuentra entre los 2 años y medio y 4 años. Explican que existen distintos factores ambientales y de estrés, como la presión comunicativa del contexto, la actitud del interlocutor, el tema de conversación o la emoción que sienta el niño mientras habla, pueden influir en su fluidez (35), (3).

Por esto, es de carácter imperativo, buscar estrategias que tengan como objetivo principal, la rehabilitación de los mecanismos necesarios para la fluidez verbal en los infantes, de ahí nace la necesidad del fonoaudiólogo como profesional integral especialista en el tratamiento de los trastornos comunicativos y del habla, objeto que solo se puede lograr cumpliendo con la expectativa de tratamiento para la mejoría de dichos problemas, realizando búsquedas, investigaciones, revisiones de las metodologías más eficientes en el mundo según el campo fonoaudiológico en el que se desea intervenir (3).

La revisión sistemática arrojó una gran cantidad de estrategias de intervención temprana de la tartamudez infantil, pero no eran respaldados por una base bibliográfica fuerte y estable por lo cual se llega a la conclusión sobre un método estandarizado llamado: Programa Lidcombe, este se define como un tratamiento conductual para niños con trastornos en la fluidez verbal (tartamudez) cuyo objetivo del programa Lidcombe es eliminar o reducir el tartamudeo (24).

Este programa utiliza herramientas que involucran una acción operante, donde son los padres directamente los que lo llevan a cabo, es decir, mediante una terapia indirecta ellos contribuyen a la mejoría de sus hijos desde casa. Las contingencias se aplican reforzando el habla fluente y corrigiendo el tartamudeo. El proceso no sólo se basa en que los padres realicen diferentes técnicas de rehabilitación, por parte del profesional de salud, es importante enseñar a los padres, es decir, involucra un proceso de aprendizaje llevado a cabo mediante visitas semanales donde se enseña a los padres a administrar el tratamiento a su hijo, a medir la gravedad de las difluencias del niño, a premiar el habla fluida y a corregir el tartamudeo (2).

En la búsqueda de la bibliografía se puede evidenciar, de cómo el programa Lidcombe ha causado un impacto en los diferentes grupos de investigación e intervención a lo largo del mundo (especialmente en Australia, los países Europeos, Asia y Norte América). La fundamentación teórica del programa Lidcombe se basa en la modificación de la conducta del tartamudeo, por esta razón las generalidades de la intervención se estructuran en 5 etapas llamadas contingencias verbales las cuales se clasifican en 3 contingencias (Stutterfree) para hablar sin tartamudear y 2 contingencias donde es seguro e indudable que el niño realice el tartamudeo (4).

Las contingencias verbales para hablar sin realizar el tartamudeo, son fundamentales para el programa Lidcombe ya que los niños por encima de todo deben disfrutar del tratamiento. Por lo tanto las contingencias verbales de los padres para hablar sin tartamudear necesitan ser sustancialmente positivos, de apoyo y agradables.

La primera contingencia verbal es la alabanza, es aquí donde se haya la matriz del tratamiento, el fonoaudiólogo orienta a los padres a elogiar a sus hijos al momento en que realiza las emisiones verbales sin las características del tartamudeo, la importancia de este modelo, se basa en que los cuidadores adapten los elogios de manera autentica y que no llegue a exagerar el cumplido a tal punto que deje de ser una motivación para el niño. La segunda contingencia verbal, se centra en la petición de la autoevaluación donde se le pide al niño que evalúe su producción verbal cuando no realiza el tartamudeo, esto puede presentarse en un enunciado, palabra o por algunas horas que no se muestren difluencias en las producciones verbales, con esto el infante concierta de como el mismo puede realizar las producciones verbales sin que manifiesten las características de la tartamudez. 
La tercera contingencia verbal es la de reconocer, el objetivo no es evaluar la fluidez verbal por esto se diferencia de las anteriores, ya que el niño tiene la capacidad de registrar las producciones verbales sin tartamudear, este es un proceso que realiza el niño de manera automática, según la orientación de sus padres y el fonoaudiólogo.

Las contingencias verbales donde el tartamudeo es indudable deben ser tratadas de forma cuidadosa ya que en este punto se comienzan a corregir los errores en la fluidez verbal de los niños y puede que reaccionen inicialmente de una manera negativa. La primera contingencia verbal para el tartamudeo indudable es reconocer, el niño tiene la capacidad de reconocer como hablar sin tartamudear, esta contingencia no es del todo evaluativa.

El padre sencillamente señala que se ha producido el tartamudeo y sigue adelante. La inferencia primordial del programa Lidcombe es que las contingencias verbales de los padres son el modelo de tratamiento activo para eliminar o reducir el habla tartamuda. Consecuentemente, cuando el fonoaudiólogo sienta que sea adecuado, es lógico para aquellas contingencias verbales de los padres que se produzcan durante las conversaciones naturales con los niños (4).

\section{CONCLUSIONES}

-En la búsqueda exhaustiva de la bibliografía para la revisión sistemática se encontró que en la actualidad existen métodos de rehabilitación para la tartamudez infantil pero no todos tienen una base teórica sólida y estable.

-El programa Lidcombe es un método de rehabilitación de la fluidez verbal de tipo conductual que tiene como objetivo disminuir o eliminar la tartamudez infantil.

- La eficacia del programa Lidcombe se evidencia en las fuentes bibliográficas y en la metodología GRA$\mathrm{DE}$, que se usó para la revisión sistemática con el fin de darle respuesta a la pregunta de investigación, del mejor método de rehabilitación de la fluidez verbal en la tartamudez infantil.

\section{REFERENCIAS BIBLIOGRÁFICAS}

1. Luis M. Las teorías de los actos de habla [Tesis]. Universidad De Oviedo (España); 2016.

2. Sangorrín J. Trastornos secundarios a déficit instrumental. Rev Neurol.2005; 41(1): S43-S46.

3. Calvo MEK, Molina MA, Gómez D. Enfoque y manejo de la tartamudez. Pediatr Aten Primaria. 2015; 17(65):e49-51. Doi.org/10.4321/S1139-76322015000100011.

4. Santos CM da C, Pimenta CA de M, Cuce MR. The PICO strategy for the research question construction and evidence search. Rev. Latino-Am.Enfermagem.2007:15(3).508-11. Doi.org/10.1590/S010411692007000300023

5. Mustafa RA, Santesso N, Brozek J, Akl EA, Walter SD, Norman G, et al. The GRADE approach is reproducible in assessing the quality of evidence of quantitative evidence syntheses. J Clin Epidemiol. 2013;66 (7):736-742.

6. Onslow M, Jones M, O'Brian S, Packman A, Menzies R, Lowe R, et al. Comparison of percentage of syllables stuttered with parent-reported severity ratings as a primary outcome measure in clinical trials of early stuttering treatment. J Speech, Lang Hear Res. 2018;61(4):811-9. Doi.org/10.1044/2017_ JSLHR-S-16-0448

7. Nippold MA. Stuttering in Preschool Children: Direct Versus Indirect Treatment. Lang Speech Hear Serv Sch. 2018; 49(1):4-12.Doi.Org/10.1044/2017_LSHSS-17-0066

8. Imeson J, Lowe R, Onslow M, Munro N, Heard R, O'Brian S, Arnott S. The Lidcombe Program and child language development: Long-term assessment. Clin Linguist Phonetics. 2018; 32(9):860-75. DOI: 10.1080/02699206.2018.1448897 
9. Unicomb R, Hewat S, Spencer E, Harrison E. Evidence for the treatment of co-occurring stuttering and speech sound disorder: A clinical case series. Int J Speech Lang Pathol. 2017; 19(3):251-64. DOI: 10.1080/17549507.2017.1293735

10. Bailey DJ, Blomgren M, DeLong C, Berggren K, Wambaugh JL. Quantification and Systematic Characterization of Stuttering-Like Disfluencies in Acquired Apraxia of Speech. Am J Speech-Language Pathol. 2017; 26(2S):641-8. Doi.Org/10.1044/2017_AJSLP-16-0108

11. Sawyer J, Matteson C, Ou H, Nagase T. The Effects of Parent-Focused Slow Relaxed Speech Intervention on Articulation Rate, Response Time Latency, and Fluency in Preschool Children Who Stutter. J Speech, Lang Hear Res. 2017;60(4):794-809. DOI: 10.1044/2016_JSLHR-S-16-0002

12. Bridgman K, Onslow M, O'Brian S, Jones M, Block S. Lidcombe Program Webcam Treatment for Early Stuttering: A Randomized Controlled Trial. J Speech, Lang Hear Res. 2016; 59(5):932-9. doi. org/10.1044/2016_JSLHR-S-15-0011

13. McCulloch J, Swift MC, Wagnitz B. Case file audit of Lidcombe program outcomes in a student-led stuttering clinic. Int J Speech Lang Pathol. 2017; 19(2):165-73. DOI: 10.3109/17549507.2016.1159336

14. Swift MC, Jones M, O'Brian S, Onslow M, Packman A, Menzies R. Parent verbal contingencies during the Lidcombe Program: Observations and statistical modeling of the treatment process. J Fluency Disord. 2016;47:13-26. DOI: 10.1016/j.jfludis.2015.12.002

15. de Sonneville-Koedoot C, Bouwmans C, Franken MC, Stolk E. Economic evaluation of stuttering treatment in preschool children: The RESTART-study. J Commun Disord. 2015;58(6):106-18. DOI: 10.1016/j.jcomdis.2015.10.006

16. de Sonneville C, Adams SA, Stolk EA, Franken MC. Perspectives of Clinicians Involved in the RESTART-Study: Outcomes of a Focus Group. Am J Speech-Language Pathol. 2015;24(4):708-16. DOI: 10.1044/2015_AJSLP-14-0215

17. Guitar B, Kazenski D, Howard A, Cousins SF, Fader E, Haskell P. Predicting Treatment Time and LongTerm Outcome of the Lidcombe Program: A Replication and Reanalysis. Am J Speech-Language Pathol. 2015;24(3):533-44. Doi.org/10.1044/2015_AJSLP-13-0156

18. Chu SY, Sakai N, Mori K. An Overview of Managing Stuttering in Japan. Am J Speech-Language Pathol. 2014;23(4):742-52. DOI: 10.1044/2014_AJSLP-13-0085

19.Arnott S, Onslow M, O'Brian S, Packman A, Jones M, Block S. Group Lidcombe Program Treatment for Early Stuttering: A Randomized Controlled Trial. J Speech, Lang Hear Res. 2014;57(5):1606-18. doi. org/10.1044/2014_JSLHR-S-13-0090

20. 'Brian S, Smith K, Onslow M. Webcam Delivery of the Lidcombe Program for Early Stuttering: A Phase I Clinical Trial. J Speech, Lang Hear Res.2014;57(3):825-30. doi.org/10.1044/2014_JSLHR-S-13-0094

21. O'Brian S, Iverach L, Jones M, Onslow M, Packman A, Menzies R. Effectiveness of the Lidcombe Program for early stuttering in Australian community clinics. Int J Speech Lang Pathol. 2013;15(6):593-603. DOI: 10.3109/17549507.2013.783112

22. Femrell L, Åvall M, Lindström E. Two-year follow-up of the Lidcombe Program in ten Swedish-speaking children. Folia Phoniatr Logop. 2012;64(5):248-53. Doi: 10.1159/000342149.

23. Packman A, Onslow M. Investigating optimal intervention intensity with the Lidcombe Program of early stuttering intervention. International Journal of Speech-Language Pathology. 2012:14(5):467-70. doi: 10.3109/17549507.2012.689861

24. Fernández A, De León M, Gambra S. Evaluación, intervención y evolución en un caso de tartamudez temprana. Bol AELFA. 2011;11(2):39-44.

25. Koushik S, Hewat S, Shenker RC, Jones M, Onslow M. North-american lidcombe program Revista Científica Signos Fónicos, 2018,4(2): 13-28. ISNN 2422-1716. 
file audit: Replication and meta-analysis. Int J Speech Lang Pathol. 2011; 13(4):301-7. DOI: 10.3109/17549507.2011.538434

26. Goodhue R, Onslow M, Quine S, O'Brian S, Hearne A. The Lidcombe Program of early stuttering intervention: mothers' experiences.J Fluency Disord. 2010; 35(1):70-84.Doi: 10.1016/j.jfludis.2010.02.002

27. Vong E, Wilson L, McAllister L, Lincoln M. Malaysian Parents'Use of Positive Reinforcement: A Descriptive Study and Implications for the Lidcombe Program. Asia Pacific J Speech, Lang Hear. 2010;13(2):87108.

28. Koushik S, Shenker R, Onslow M. Follow-up of 6-10-year-old stuttering children after Lidcombe Program treatment: A Phase I trial. J Fluency Disord.2009;34(4):279-90.DOI: 10.1016/j.jfludis.2009.11.001

29. Bakhtiar M, Packman A. Intervention with the lidcombe program for a bilingual school-age child who stutters in Iran. Folia Phoniatr Logop. 2009; 61(5):300-4. Doi: 10.1159/000241880.

30. Miller B, Guitar B. Long-Term Outcome of the Lidcombe Program for Early Stuttering Intervention. Am J Speech-Language Pathol. 2008; 18(1):42-9. DOI:10.1044/1058-0360(2008/06-0069).

31. Lim VPC, Lincoln M, Chan YH, Onslow M. Stuttering in English-Mandarin Bilingual Speakers: The Influence of Language Dominance on Stuttering Severity. J Speech, Lang Hear Res. 2008; 51(6):1522-37. Doi: 10.1044/1092-4388(2008/07-0054).

32.Jones M, Onslow M, Packman A, O'Brian S, Hearne A, Williams S, Ormond T, Schwarz I.. Extended follow-up of a randomized controlled trial of the Lidcombe program of early stuttering intervention. Int J Lang Commun Disord. 2008; 43(6):649-61. DOI: 10.1080/13682820801895599

33. Lattermann C, Euler HA, Neumann K. A randomized control trial to investigate the impact of the Lidcombe Program on early stuttering in German-speaking preschoolers. J Fluency Disord. 2008;33(1):5265. DOI: 10.1016/j.jludis.2007.12.002

34. Lewis L, Packman A, Onslow M, Simpson JM.A Phase II Trial of Telehealth Delivery of the Lidcombe Program of Early Stuttering Intervention. American Journal of Speech-Language Pathology.2008; 17(2):139-49. DOI: 10.1044/1058-0360(2008/014).

35. Coll M, Aguado G, Fernández A, Gambra S, Perelló E, VILA JM. Trastornos del habla y de la voz. $1^{\text {a }}$ Edición. Barcelona: Editorial UOC; 2014. 